\title{
Ranking Websites: A Probabilistic View
}

\author{
Ying Bao, Guang Feng, Tie-Yan Liu, Zhi-Ming Ma, and Ying Wang
}

Abstract. In this paper we suggest evaluating the importance of a website with the mean frequency of visiting the website for the Markov chain on the Internet graph describing random surfing. We show that this mean frequency is equal to the sum of the PageRanks of all the webpages in that website (hence referred to as PageRankSum), and we propose a novel algorithm, AggregateRank, based on the theory of stochastic complement to calculate the rank of a website. The AggregateRank algorithm gives a good approximation of the PageRankSum accurately, while the corresponding computational complexity is much lower than PageRankSum. By constructing return-time Markov chains restricted to each website, we describe also the probabilistic relation between PageRank and AggregateRank. The complexity of the AggregateRank algorithm, the error bound of the estimation, and the experiments are discussed at the end of the paper.

\section{Introduction}

The design of web search engines has become a focus of the research on web search and mining. One popular approach is to calculate static ranks by exploiting the hyperlink structure of the web. Researchers have made great progresses on link-analysis models and algorithms since 1998, such as HITS and PageRank [Kleinberg 99, Brin et al. 99]. Nowadays, PageRank has emerged as a popular link-analysis model, mostly due to its query independence, using only the web graph structure, and Google's huge business success.

While a webpage, an essential element of the web, is still a focus of the research on web search and mining, in recent years many researchers have realized that

(C) A K Peters, Ltd.

I542-795I/06 \$0.50 per page 
the website, another element of the web, plays a more and more important role in web search and mining applications [Despeyroux 04, Davulcu et al. 04, Lerman et al. 04, Lei et al. 04, Qin et al. 05]. Compared to an individual webpage, a website can sometimes provide plenty of semantic information in the following aspects. First, webpages in the same website may be authored by the same person or organization. Second, there might be high correlations between webpages in the same website, in terms of content, page layout, and hyperlinks. Third, from the topological point of view, websites contain a higher density of internal hyperlinks (about $75 \%$ according to [Henzinger et al. 03]) and a lower density of external links [Girvan and Newman 02]. These properties make websites semantically important to understanding the whole picture of the web. Actually, the ranking of websites has been a key technical component in many commercial search engines. On the one hand, in the service part, it can help define a more reasonable rank for webpages because those pages from important websites tend to be important as well. On the other hand, in the crawler part, it can help crawl webpages from those important websites first, or help determine a quota (number of webpages) for each website in the index according to its rank. In this way, with the same size of index in total, search engines can achieve the best tradeoff between index coverage and index quality.

In the literature of website ranking, researchers used to describe the interconnectivity among websites with a so-called hostgraph in which the nodes denote websites and the edges denote links between websites (there will be an edge between two websites if and only if there is a hyperlink from a webpage in one website to a webpage in the other) and then adopt a random-walk model in the hostgraph to calculate the website ranking [Bharat et al. 01, Dill et al. 01]. However, we want to point out that a random walk over such a graph does not reasonably reflect the browsing behavior of web surfers. In this paper we shall propose a reasonable evaluation for ranking the websites. Namely, we suggest evaluating the importance of a website by the mean frequency of visits to the site made by a Markov chain on the Internet Graph that models random surfing. We shall prove (see Theorem 3.3) that this mean frequency is equal to the sum of the PageRanks of all the webpages in that website (denoted by PageRankSum for ease of reference).

However, it is clear that using PageRankSum to calculate the ranks of websites is not yet a feasible solution, especially for those applications that only care about webpages. The reason is that the number of webpages is much larger than the number of websites. Therefore, it is much more complex to rank webpages than to rank websites, and it is almost impossible for small research groups or companies to afford such expensive computations. To tackle these aforementioned problems, we propose a novel algorithm for calculating the rank of a 
website - based on the theory of stochastic complement [Meyer 89] - that can approximate the PageRankSum accurately but whose computational complexity is much lower than PageRankSum and only a little higher than previous HostRank algorithms [Bharat et al. 01, Dill et al. 01]. We name this algorithm AggregateRank. Experiments demonstrated the effectiveness and efficiency of this algorithm.

Since PageRank reflects the mean frequency of visits to webpages (see Section 2) and AggregateRank reflects the mean frequency of visits to websites, both algorithms are closely related. By constructing return-time Markov chains restricted to each website, we may formulate the relation between PageRank and AggregateRank as follows. Suppose that AggregateRank is $\xi=\left(\xi_{1}, \xi_{2}, \cdots, \xi_{N}\right)$, that $P_{S_{i}}(\alpha)$ denotes the transition matrix of the return-time Markov chain for website $S_{i}$ (for $i=1,2, \cdots, N$ ), and that the stationary distribution of $P_{S_{i}}(\alpha)$ is $\pi_{S_{i}}(\alpha), i=1,2, \cdots, N$. Then,

$$
\text { PageRank }=\left(\xi_{1} \pi_{S_{1}}(\alpha), \xi_{2} \pi_{S_{2}}(\alpha), \cdots, \xi_{N} \pi_{S_{N}}(\alpha)\right) .
$$

The rest of this paper is organized as follows. In Section 2 we briefly review the probabilistic meaning of PageRank and explain that PageRank reflects the mean frequency of webpage visits. In Section 3 we explore how to reasonably rank websites with the mean frequency of visits as well, and we describe our AggregateRank algorithm. In Section 4 we describe the probabilistic relation between PageRank and AggregateRank. In Section 5 we discuss the complexity and the error bound of the AggregateRank algorithm and report on experiments with some real web-graph data.

\section{Explaining PageRank with Markov Chain}

How to rank webpages has been investigated widely, and one of the most famous algorithms is called PageRank [Brin et al. 99, Langville and Meyer 04], which was proposed by Brin and Page in 1998 and is used by the Google search engine. The probabilistic meaning of PageRank has been explained in the literature (see, e.g., [Langville and Meyer 04]). For the purpose of our further discussion, we briefly review the probabilistic meaning of PageRank and provide a more explicit explanation via the ergodic theorem of Markov chains.

Consider the hyperlink structure of webpages on a network as a directed graph $G=(V(G), E(G))$ [Bao and Liu 06]. A vertex $i \in V(G)$ of the graph represents a webpage, and a directed edge $\overrightarrow{i j} \in E(G)$ represents a hyperlink from page $i$ to page $j$. Let $B$ be the adjacent matrix of $G$ and $b_{i}$ be the sum of the $i$ th row of $B$. Let $D$ be the diagonal matrix with diagonal entry $b_{i}$ (If $b_{i}=0$, then we 
normalize $b_{i}=n$, the cardinal number of $V(G)$, and change all entries of the $i$ th row of $B$ to 1 ). Now, we construct a stochastic matrix $P=D^{-1} B$.

When a surfer browses on the Internet, he may choose the next page by randomly clicking on one of the links in the current page. This happens with a large probability $\alpha$, which means that he randomly walks on $G$ with transition probability $P$. Sometimes, however, he may open a new page randomly-not following the hyperlinks. This occurs with a small probability $(1-\alpha)$, which means that he randomly walks on $G$ with transition probability $\frac{1}{n} e e^{T}$, where $e$ is a column vector of all ones. So, the transition matrix that describes the random surfer behavior is formulated as

$$
P(\alpha)=\alpha P+(1-\alpha) \frac{1}{n} e e^{T} .
$$

The random surfer model can be formally described by a Markov chain $\left\{X_{k}\right\}_{k \geq 0}$. The evolution of the Markov chain represents the surfing behavior of a random surfer from one webpage to another. So, the transition matrix of $\left\{X_{k}\right\}_{k \geq 0}$ is $P(\alpha)$, which is an irreducible stochastic matrix on a finite state space that has a unique stationary distribution.

PageRank algorithms use the stationary distribution of $P(\alpha)$ (denoted by $\pi(\alpha)$, which satisfies $\pi(\alpha) P(\alpha)=\pi(\alpha)$ with $\pi(\alpha) e=1)$ to evaluate the importance of webpages. That is to say, webpages are ranked according to their value in $\pi(\alpha)$.

Now we will explain the probabilistic meaning for PageRank more explicitly. We learn from the ergodic theorem on Markov chains (see, e.g., [Qian and Gong 97]) that

$$
\begin{aligned}
\pi^{i}(\alpha) & =\lim _{n \rightarrow \infty} \frac{1}{n} \sum_{k=0}^{n-1} p_{i i}^{(k)}(\alpha) \\
& =\left(\sum_{n=1}^{\infty} n f_{i i}(n)\right)^{-1}
\end{aligned}
$$

where $\pi^{i}(\alpha)$ is the $i$ th entry of $\pi(\alpha), p_{i i}^{(k)}(\alpha)$ is the $i i$ th entry of the $k$-step transition matrix $P^{k}(\alpha)$, and $f_{i i}(n)$ is the probability of first returning to page $i$ in $n$ steps after starting from page $i$. Expression (2.3) is equal to the mean frequency of visits to page $i$. The more important a webpage is, the higher the frequency that it will be visited. So, the ergodic theorem on Markov chains shows that the stationary distribution $\pi(\alpha)$ of $P(\alpha)$ is a very suitable candidate for ranking webpages. 


\section{Probabilistic View of Ranking Websites}

PageRank has been proved to be successful in web search. Actually, ranking is important not only for webpages but also for websites in many applications. There are two approaches in the literature of website ranking. However, we will show that the traditional approaches on calculating website ranks are not reasonable because they lose some transition information of the random surfer (see Section 3.1). To tackle this problem, we will investigate the real transition probability between websites in Section 3.2, and then, based on the investigation, propose a novel algorithm for website ranking in Section 3.3.

\section{I. Traditional Approaches to Calculating Website Ranks}

In the literature of website ranking, people used to apply those technologies proposed for ranking webpages to the ranking of websites. For example, the famous PageRank algorithm was used to rank websites in the works [Eiron et al. 04, $\mathrm{Wu}$ and Aberer 04]. In order to apply PageRank to the ranking of websites, a hostgraph was constructed in these works. In the hostgraph, the nodes denote websites, and there is an edge between two nodes if there is a hyperlink from a webpage in one website to a webpage in the other. According to different definitions of the edge weights, there are two categories of hostgraphs are used in the literature. In the first category, the weight of an edge between two websites is defined by the number of hyperlinks between the two sets of webpages in these sites [Bharat et al. 01]. In the second category, the weight of any edge is simply set to 1 [Dill et al. 01]. For the sake of clarity, we refer to the two categories as weighted hostgraph and naïve hostgraph, respectively. Figures 1 and 2 show how these two categories of hostgraphs can be constructed.

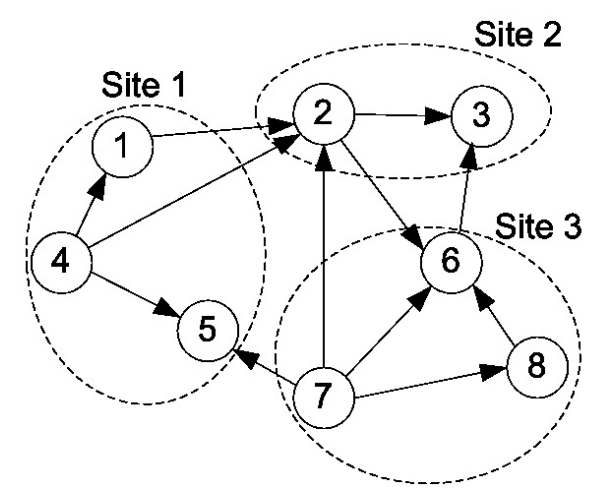

Figure I. Illustration of a web graph with several websites. 


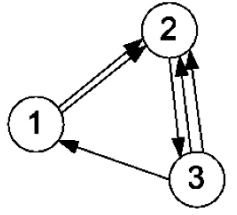

(a)

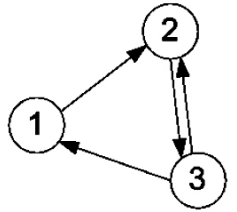

(b)

Figure 2. Illustrations of the (a) weighted hostgraph and (b) naïve hostgraph of the web graph in Figure 1.

After constructing the hostgraph, a similar random walk was conducted in both works. That is, a random surfer was supposed to jump between websites following the edges, with a probability of $\alpha$, or jump to a random website, with a probability of $1-\alpha$. In such a way, one can obtain the HostRank, which is used to describe the importance of the websites.

At first glance, the above random-walk model over the hostgraph seems to be a natural extension of the PageRank algorithm. However, we want to point out that it is actually not as reasonable for sites as it is for pages because it is not in accordance with the browsing behavior of web surfers. As we know, real-world web surfers usually have two basic ways to access the web. One is to type the URL in the address bar of the web browser. The other is to click on a hyperlink in the currently loaded page. These two methods can be described well by the parameter $\alpha$ used in PageRank. That is, with a probability of $1-\alpha$, the web users visit a random webpage by inputting its URL (using a favorites folder can be considered as a shortcut to typing URLs), and, with a probability of $\alpha$, they visit a web page by clicking on a hyperlink.

Nevertheless, for the random walk in the hostgraph, we can hardly find the same evident correlation between the random-walk model and real-world user behaviors. For example, even if there is an edge between two websites A and B in the hostgraph, when a web surfer visits a page in website A, he may not be able to jump to website B because the hyperlink to website B may exist on another page in website A, which may be unreachable from the page that he is currently visiting. In other words, the hostgraph is only a kind of approximation to the web graph: it loses much transition information, especially for the naïve hostgraph. As a result, we argue that the rank values derived from the aforementioned hostgraph are not convincing enough.

\subsection{Transition Probability between Websites}

Motivated by the probabilistic explanation of PageRank discussed in Section 2, we propose that a reasonable way to describe the importance of a website should 
be the mean frequency with which users visit it. Actually, the Markov chain $\left\{X_{k}\right\}_{k \geq 0}$ also implies the transition between websites. We should do a little deduction to expose it.

Suppose that there are $N$ total websites in the web. Each webpage belongs to some determinate website, so we rearrange the transition matrix $P(\alpha)$ and partition it into $N \times N$ blocks according to the $N$ websites. Then, it has the following form:

$$
P(\alpha)=\left(\begin{array}{cccc}
P_{11}(\alpha) & P_{12}(\alpha) & \cdots & P_{1 N}(\alpha) \\
P_{21}(\alpha) & P_{22}(\alpha) & \cdots & P_{2 N}(\alpha) \\
\vdots & \vdots & \ddots & \vdots \\
P_{N 1}(\alpha) & P_{N 2}(\alpha) & \cdots & P_{N N}(\alpha)
\end{array}\right)
$$

where the elements in each diagonal block denote the transition probabilities between webpages in the same website and the elements of each off-diagonal block denote the transition probabilities between webpages in different websites. The diagonal blocks $P_{i i}(\alpha)$ are square and of order $n_{i}$, for $i=1,2, \cdots, N$, and $n=\sum_{i=1}^{N} n_{i}$. The stationary distribution $\pi(\alpha)$, known as the PageRank vector, is given by

$$
\pi(\alpha) P(\alpha)=\pi(\alpha) \text { with } \pi(\alpha) e=1 .
$$

Let $\pi(\alpha)$ be partitioned conformally with $P(\alpha)$, i.e.,

$$
\pi(\alpha)=\left(\pi_{1}(\alpha), \pi_{2}(\alpha), \cdots, \pi_{N}(\alpha)\right)
$$

where $\pi_{i}(\alpha)$ is a row vector of length $n_{i}$.

Till now, we just get a rearranged PageRank. However, this process is necessary to describe the next part plainly.

We now turn our attention to the mean frequency that a random surfer visits the website $S_{j}$ (for any fixed $j=1, \cdots, N$ ). Since we are interested in the situation that the surfing Markov chain $\left\{X_{k}\right\}_{k \geq 0}$ has run a long time, we may assume that $\left\{X_{k}\right\}_{k \geq 0}$ starts from the stationary probability $\pi(\alpha)$. Then, the one-step transition probability from the website $S_{i}$ to the website $S_{j}$ is defined by

$$
c_{i j}(\alpha)=\operatorname{Pr}_{\pi(\alpha)}\left\{X_{m+1} \in S_{j} \mid X_{m} \in S_{i}\right\} .
$$

The $k$-step transition probability from the website $S_{i}$ to the website $S_{j}$ is defined by

$$
c_{i j}^{(k)}(\alpha)=P r_{\pi(\alpha)}\left\{X_{m+k} \in S_{j} \mid X_{m} \in S_{i}\right\}
$$


Recall that $\|\cdot\|_{1}$ is the 1-norm of a vector, i.e., the sum of all entries of a vector.

Theorem 3.I. From a website $S_{i}$ to another website $S_{j}$, the one-step transition probability is $c_{i j}(\alpha)=\frac{\pi_{i}(\alpha)}{\left\|\pi_{i}(\alpha)\right\|_{1}} P_{i j}(\alpha) e$.

Proof. By the properties of conditional probability, we have

$$
\begin{aligned}
c_{i j}(\alpha) & =\operatorname{Pr}_{\pi(\alpha)}\left\{X_{m+1} \in S_{j} \mid X_{m} \in S_{i}\right\} \\
& =\frac{\operatorname{Pr}_{\pi(\alpha)}\left\{X_{m+1} \in S_{j}, X_{m} \in S_{i}\right\}}{\operatorname{Pr}_{\pi(\alpha)}\left\{X_{m} \in S_{i}\right\}} \\
& =\frac{\sum_{t \in S_{j}} \sum_{l \in S_{i}} \operatorname{Pr}_{\pi(\alpha)}\left\{X_{m+1}=t, X_{m}=l\right\}}{\sum_{l \in S_{i}} \operatorname{Pr}_{\pi(\alpha)}\left\{X_{m}=l\right\}} \\
& =\frac{\sum_{t \in S_{j}} \sum_{l \in S_{i}} \operatorname{Pr}_{\pi(\alpha)}\left\{X_{m}=l\right\} P r_{\pi(\alpha)}\left\{X_{m+1}=t \mid X_{m}=l\right\}}{\sum_{l \in S_{i}} \operatorname{Pr}_{\pi(\alpha)}\left\{X_{m}=l\right\}} \\
& =\frac{\sum_{t \in S_{j}} \sum_{l \in S_{i}} \pi^{l}(\alpha) p_{l t}(\alpha)}{\sum_{l \in S_{i}} \pi^{l}(\alpha)} \\
& =\frac{\pi_{i}(\alpha) P_{i j}(\alpha) e}{\left\|\pi_{i}(\alpha)\right\|_{1}} \\
& =\frac{\pi_{i}(\alpha)}{\left\|\pi_{i}(\alpha)\right\|_{1}} P_{i j}(\alpha) e,
\end{aligned}
$$

where $\pi^{l}(\alpha)$ is the $l$ th entry of $\pi(\alpha), p_{l t}(\alpha)$ is the $l t$ th entry of $P(\alpha)$, and $e$ is a column vector of all ones of which the dimension depends on the corresponding context.

Theorem 3.2. For two websites $S_{i}$ and $S_{j}$, the $k$-step transition probability is

$$
c_{i j}^{(k)}(\alpha)=\frac{\pi_{i}(\alpha)}{\left\|\pi_{i}(\alpha)\right\|_{1}} P_{i j}^{(k)}(\alpha) e
$$

where $P_{i j}^{(k)}(\alpha)$ is the ijth block of the $k$-step transition matrix $P^{k}(\alpha)$.

Proof. By the properties of conditional probability, we have

$$
\begin{aligned}
c_{i j}^{(k)}(\alpha) & =\operatorname{Pr}_{\pi(\alpha)}\left\{X_{m+k} \in S_{j} \mid X_{m} \in S_{i}\right\} \\
& =\frac{\operatorname{Pr}_{\pi(\alpha)}\left\{X_{m+k} \in S_{j}, X_{m} \in S_{i}\right\}}{\operatorname{Pr}_{\pi(\alpha)}\left\{X_{m} \in S_{i}\right\}} \\
& =\frac{\sum_{t \in S_{j}} \sum_{l \in S_{i}} \operatorname{Pr}_{\pi(\alpha)}\left\{X_{m+k}=t, X_{m}=l\right\}}{\sum_{l \in S_{i}} \operatorname{Pr}_{\pi(\alpha)}\left\{X_{m}=l\right\}}
\end{aligned}
$$




$$
\begin{aligned}
& =\frac{\sum_{t \in S_{j}} \sum_{l \in S_{i}} \operatorname{Pr}_{\pi(\alpha)}\left\{X_{m}=l\right\} \operatorname{Pr}_{\pi(\alpha)}\left\{X_{m+k}=t \mid X_{m}=l\right\}}{\sum_{l \in S_{i}} \operatorname{Pr}_{\pi(\alpha)}\left\{X_{m}=l\right\}} \\
& =\frac{\sum_{t \in S_{j}} \sum_{l \in S_{i}} \pi^{l}(\alpha) p_{l t}^{(k)}(\alpha)}{\sum_{l \in S_{i}} \pi^{l}(\alpha)} \\
& =\frac{\pi_{i}(\alpha) P_{i j}^{(k)}(\alpha) e}{\left\|\pi_{i}(\alpha)\right\|_{1}} \\
& =\frac{\pi_{i}(\alpha)}{\left\|\pi_{i}(\alpha)\right\|_{1}} P_{i j}^{(k)}(\alpha) e,
\end{aligned}
$$

where $p_{l t}^{(k)}(\alpha)$ is the ltth entry of $P^{k}(\alpha)$.

We have assumed that $\pi(\alpha)$ is the initial distribution of the webpagesurfing Markov chain $\left\{X_{k}\right\}_{k \geq 0}$. We assume that a surfer is browsing on some website $S_{i}$ at time $m$, and we will calculate the number of visits to the website $S_{j}$ after time $m$. Let $N_{j}(n)$ denote the number of $\left\{X_{k}\right\}_{k \geq 0}$ visits to the website $S_{j}$ during the $n$ times $\{m+1, m+2, \cdots, m+n\}$. Then, we get the following conclusion.

Theorem 3.3. The 1-norm of the initial distribution of the Markov chain surfing website $S_{j}$ is

$$
\left\|\pi_{j}(\alpha)\right\|_{1}=E\left(\lim _{n \rightarrow \infty} \frac{N_{j}(n)}{n}\right) .
$$

Proof. By definition, we know that $E\left(\lim _{n \rightarrow \infty} \frac{N_{j}(n)}{n}\right)$ is the mean frequency of visits to the website $S_{j}$. Hence, by the dominated convergence theorem (e.g., [Kallenberg 97]) and the ergodic theorem on Markov chains, we get

$$
\begin{aligned}
E\left(\lim _{n \rightarrow \infty} \frac{N_{j}(n)}{n}\right) & =E\left(\lim _{n \rightarrow \infty} \frac{\sum_{k=1}^{n} \mathbf{1}_{\left\{X_{m+k} \in S_{j}\right\}}}{n}\right) \\
& =\lim _{n \rightarrow \infty} E\left(\frac{\sum_{k=1}^{n} \mathbf{1}_{\left\{X_{m+k} \in S_{j}\right\}}}{n}\right) \\
& =\lim _{n \rightarrow \infty} \frac{1}{n} \sum_{k=1}^{n} c_{i j}^{(k)}(\alpha) \\
& =\lim _{n \rightarrow \infty} \frac{1}{n} \sum_{k=1}^{n} \frac{\pi_{i}(\alpha)}{\left\|\pi_{i}(\alpha)\right\|_{1}} P_{i j}^{(k)}(\alpha) e \\
& =\frac{\pi_{i}(\alpha)}{\left\|\pi_{i}(\alpha)\right\|_{1}}\left(\lim _{n \rightarrow \infty} \frac{1}{n} \sum_{k=1}^{n} P_{i j}^{(k)}(\alpha)\right) e
\end{aligned}
$$




$$
\begin{aligned}
& =\frac{\pi_{i}(\alpha)}{\left\|\pi_{i}(\alpha)\right\|_{1}}\left(e \pi_{j}(\alpha)\right) e \\
& =\left\|\pi_{j}(\alpha)\right\|_{1},
\end{aligned}
$$

where

$$
\mathbf{1}_{\left\{X_{m+k} \in S_{j}\right\}}= \begin{cases}1, & \text { when } X_{m+k} \in S_{j} \\ 0, & \text { otherwise. }\end{cases}
$$

From the deduction above, we know that $\left\|\pi_{j}(\alpha)\right\|_{1}$ is the mean frequency of visits to $S_{j}$. Hence, the probability vector

$$
\left(\left\|\pi_{1}(\alpha)\right\|_{1},\left\|\pi_{2}(\alpha)\right\|_{1}, \cdots,\left\|\pi_{N}(\alpha)\right\|_{1}\right)
$$

is a suitable candidate for ranking the importance of websites.

As aforementioned, $c_{i j}(\alpha)$ represents the transition probability between websites. By virtue of Theorem 3.1, we see that the $N \times N$ matrix $C(\alpha)=\left(c_{i j}(\alpha)\right)$ is equal to the coupling matrix specified in Theorem 4.1 of [Meyer 89]. It follows that $C(\alpha)$ is an irreducible stochastic matrix; thus, it possesses a unique stationary probability vector, denoted by $\xi(\alpha)$, i.e.,

$$
\xi(\alpha) C(\alpha)=\xi(\alpha) \text { with } \xi(\alpha) e=1 .
$$

One can easily verify that if we define

$$
\xi(\alpha)=\left(\left\|\pi_{1}(\alpha)\right\|_{1},\left\|\pi_{2}(\alpha)\right\|_{1}, \cdots,\left\|\pi_{N}(\alpha)\right\|_{1}\right),
$$

then $\xi(\alpha)$ is a solution of (3.6).

One may have realized that the above computation can also be regarded as being carried out with a certain hostgraph. However, the edge weight of this new hostgraph is not decided heuristically as in previous works [Bharat et al. 01, Dill et al. 01] but is determined by the sophisticated formulation in Theorem 3.1. Besides, the transition probability from $S_{i}$ to $S_{j}$ actually summarizes all the cases in which the random surfer jumps from any webpage in $S_{i}$ to any webpage in $S_{j}$ within one-step transition. Therefore, the transition in this new hostgraph is in accordance with the real behavior of web surfers. In this regard, the socalculated rank from the coupling matrix $C(\alpha)$ will be more reasonable than the one in those previous works.

Based on the above discussions, the direct approach to computing the AggregateRank $\xi(\alpha)$ is to accumulate PageRank values (denoted by PageRankSum). However, this approach is unfeasible because the computation of PageRank is not a trivial task when the number of webpages is as large as several billions. Therefore, efficient computation becomes a significant problem. In the next subsection, we will propose an approximate algorithm for this purpose, which can be much more efficient than PageRankSum with very little accuracy loss. 


\subsection{The AggregateRank Algorithm}

As aforementioned, the coupling matrix $C(\alpha)$, with

$$
c_{i j}(\alpha)=\frac{\pi_{i}(\alpha)}{\left\|\pi_{i}(\alpha)\right\|_{1}} P_{i j}(\alpha) e,
$$

represents the transition probability from one website to another in terms of a random surfer model; its stationary distribution $\xi(\alpha)$, which is equal to PageRankSum, is regarded as a reasonable rank of websites. It is clear that the construction of the coupling matrix asks for the calculation of PageRank over the whole web graph. To avoid this time-consuming step, it is necessary to invent a new method for constructing the coupling matrix $C(\alpha)$. Fortunately, the theory of stochastic complement [Meyer 89] gives a good solution to form $C(\alpha)$ without PageRank values. Intuitively, the stochastic complement of a diagonal block $P_{i i}(\alpha)$ in (3.1) represents the transition matrix for the return-time Markov chain (see Section 4) restricted to the webpages of the $i$ th website. To illustrate this, we take a simple web graph as an example. Suppose that the web graph contains only two websites; the transition probability matrix of this web graph (rearranged according to website information) can be denoted by

$$
P(\alpha)=\left(\begin{array}{cc}
P_{11}(\alpha) & P_{12}(\alpha) \\
P_{21}(\alpha) & P_{22}(\alpha)
\end{array}\right)
$$

and its stationary distribution is $\pi(\alpha)=\left(\pi_{1}(\alpha), \pi_{2}(\alpha)\right)$. For each diagonal block in $P(\alpha)$, we can calculate its stochastic complement. For example, the stochastic complement of $P_{11}(\alpha)$ is calculated as follows (see also (4.15) below):

$$
S_{11}(\alpha)=P_{11}(\alpha)+P_{12}(\alpha)\left(I-P_{22}(\alpha)\right)^{-1} P_{21}(\alpha) .
$$

The stochastic complement is also a stochastic matrix, each row of which sums up to 1 . It can be proved that $\pi_{1}(\alpha) /\left\|\pi_{1}(\alpha)\right\|_{1}$ is the unique stationary probability vector for the stochastic complement $S_{11}(\alpha)$, i.e.,

$$
\frac{\pi_{1}(\alpha)}{\left\|\pi_{1}(\alpha)\right\|_{1}} S_{11}(\alpha)=\frac{\pi_{1}(\alpha)}{\left\|\pi_{1}(\alpha)\right\|_{1}} \text { with } \frac{\pi_{1}(\alpha)}{\left\|\pi_{1}(\alpha)\right\|_{1}} e=1
$$

Generally, $\pi_{i}(\alpha) /\left\|\pi_{i}(\alpha)\right\|_{1}$ is the unique stationary distribution for the stochastic complement $S_{i i}(\alpha)$, i.e.

$$
\frac{\pi_{i}(\alpha)}{\left\|\pi_{i}(\alpha)\right\|_{1}} S_{i i}(\alpha)=\frac{\pi_{i}(\alpha)}{\left\|\pi_{i}(\alpha)\right\|_{1}} \text { with } \frac{\pi_{i}(\alpha)}{\left\|\pi_{i}(\alpha)\right\|_{1}} e=1 .
$$

Apparently, the computation of the stationary distribution of each $S_{i i}(\alpha)$ will be cheaper than that of the PageRanks directly because the dimension of each 
$S_{i i}(\alpha)$ is very small and equal to the number of pages in the site. (We will discuss this in more detail in Section 4.) However, it is time-consuming to compute the exact stochastic complement since we should compute an inverse matrix for $I-P_{i i}(\alpha)$. As we know, the computation for inverse matrix is very expensive - sometimes even more expensive than PageRank. Thus, we prefer an approximate approach to get the stationary distribution of each stochastic complement instead. According to Cho and Meyer, we can find an efficient approximate method [Cho and Meyer 06]. It does not use (3.9) to aggregate the stochastic complement directly. Instead, it only modifies each diagonal block $P_{i i}(\alpha)$ by a little to get a new matrix with the same dimension as $S_{i i}(\alpha)$. The details are given as follows.

For the first step, we modify the original diagonal block $P_{i i}(\alpha)$ to be a transition probability matrix. It is clear that the sum of each row in the original diagonal block $P_{i i}(\alpha)$ is always less than 1 . To make it a transition probability matrix, we simply adjust the diagonal elements of $P_{i i}(\alpha)$ (add or subtract by a small value) to make the sum of each row equal to 1 . Letting $P_{i i}^{*}(\alpha)$ denote the matrix after adjustment, we can calculate its stationary distribution $u_{i}(\alpha)$ as

$$
u_{i}(\alpha) P_{i i}^{*}(\alpha)=u_{i}(\alpha) \text { with } u_{i}(\alpha) e=1 .
$$

According to [Cho and Meyer], we can prove that (see also (5.5) below)

$$
\frac{\pi_{i}(\alpha)}{\left\|\pi_{i}(\alpha)\right\|_{1}} \approx u_{i}(\alpha)
$$

From the description above, $P_{i i}^{*}(\alpha)$ is very easy to get from $P_{i i}(\alpha)$. Moreover, it can even be stored sparsely like the original $P(\alpha)$. Thus, the formulation in (3.13) means that we can get each $\pi_{1}(\alpha) /\left\|\pi_{1}(\alpha)\right\|_{1}$ very efficiently.

Utilizing the result of (3.13), we can obtain an approximate coupling matrix $C^{*}(\alpha)$ :

$$
\left(C^{*}(\alpha)\right)_{i j}=u_{i}(\alpha) P_{i j}(\alpha) e .
$$

Consequently, the stationary distribution $\xi^{*}(\alpha)$ of the approximate coupling matrix can be regarded as a good approximation to $\xi(\alpha)$. We name the aforementioned algorithm the AggregateRank algorithm, whose detailed algorithm flow is shown as follows:

1. Divide the $n \times n$ matrix $P(\alpha)$ into $N \times N$ blocks according to the $N$ sites.

2. Construct the stochastic matrix $P_{i i}^{*}(\alpha)$ for $P_{i i}(\alpha)$ by changing the diagonal elements of $P_{i i}(\alpha)$ so that each row sums up to 1 .

3. Determine $u_{i}(\alpha)$ from

$$
u_{i}(\alpha) P_{i i}^{*}(\alpha)=u_{i}(\alpha) \text { with } u_{i}(\alpha) e=1
$$


4. Form an approximation $C^{*}(\alpha)$ to the coupling matrix $C(\alpha)$ by evaluating

$$
\left(C^{*}(\alpha)\right)_{i j}=u_{i}(\alpha) P_{i j}(\alpha) e .
$$

5. Determine the stationary distribution of $C^{*}(\alpha)$, and denote it as $\xi^{*}(\alpha)$, i.e.,

$$
\xi^{*}(\alpha) C^{*}(\alpha)=\xi^{*}(\alpha) \text { with } \xi^{*}(\alpha) e=1
$$

To sum up, the proposed algorithm improves the efficiency in the following ways. First, it uses an easy-to-construct sparse matrix to replace the stochastic complement for approximating $\pi_{i}(\alpha) /\left\|\pi_{i}(\alpha)\right\|_{1}$ instead of the whole transition probability matrix. Second, this algorithm is much easier to implement in parallel than PageRankSum. The reason is that, for PageRank, if we want to implement it in parallel, we must take care of the information exchange between different servers since there are hyperlinks whose sources and destinations are not in the same server. For our method, we do not need the exchange of information if we put a website at most in one sever; then, the computations over $P_{i i}^{*}(\alpha)$ are done for each particular website and are independent of the rest of the web graph.

\section{Probabilistic Relation between PageRank and AggregateRank}

As is known, PageRank is carried out with a web graph, where each vertex represents a webpage, while AggregateRank is done with a hostgraph, where each vertex represents a website. If we can discover how the Markov chain $\left\{X_{k}\right\}_{k \geq 0}$ evolves when restricted to webpages of only one website, we can reveal the probability relation between PageRank and AggregateRank.

We shall introduce return times to construct a new Markov chain that describes the random surfing behavior on the pages of some fixed website $A$. Assume a starting state in website $A$, i.e., $X_{0} \in A$. The variable

$$
\tau_{A}:=\min \left\{n \geq 1 ; X_{n} \in A\right\}
$$

is called the first return time on $A$. In order to distinguish different return times, we write $\tau_{A}(k)$ for the time of the $k$ th visit to $A$; these are defined inductively by

$$
\begin{aligned}
\tau_{A}(1) & :=\tau_{A}, \\
\tau_{A}(k) & :=\min \left\{n>\tau_{A}(k-1) ; X_{n} \in A\right\} .
\end{aligned}
$$

It is clear that the variables $\tau_{A}(k)$ are stopping times for $X$. 
We construct a new stochastic process $\left\{\phi_{k}\right\}_{k \geq 0}$ as follows:

$$
\begin{aligned}
\phi_{0} & :=X_{0}, \\
\phi_{k} & :=X_{\tau_{A}(k)} .
\end{aligned}
$$

Then, we can prove that $\left\{\phi_{k}\right\}_{k \geq 0}$ is a time-homogeneous Markov chain, furthermore, an ergodic chain (see [Meyer 89]).

We now turn our attention to the transition probability matrix of $\left\{\phi_{k}\right\}_{k \geq 0}$. Assume that the transition probability matrix $P(\alpha)$ is permuted and repartitioned so that

$$
P(\alpha)=\underset{\tilde{A}}{A} \begin{gathered}
A \\
\tilde{A}
\end{gathered}\left(\begin{array}{cc}
\tilde{P}_{11}(\alpha) & \tilde{P}_{12}(\alpha) \\
\tilde{P}_{21}(\alpha) & \tilde{P}_{22}(\alpha)
\end{array}\right),
$$

where $\tilde{P}_{11}(\alpha)$ denotes the transition probabilities between webpages of website $A$. Let the stationary distribution $\pi(\alpha)$ be partitioned conformally with $P(\alpha)$, i.e.,

$$
\pi(\alpha)=\left(\pi_{1}(\alpha), \pi_{2}(\alpha)\right) .
$$

Assume that website $A$ is composed of webpages $\left\{a_{1}, a_{2}, \cdots, a_{m}\right\}$. For the new Markov chain $\left\{\phi_{k}\right\}_{k \geq 0}$, the one-step transition probability of moving from $a_{k}$ to $a_{j}$ is the probability in the original chain $\left\{X_{k}\right\}_{k \geq 0}$ of moving directly from $a_{k}$ to $a_{j}$ plus the probability of moving directly from $a_{k}$ to some state in $\tilde{A}$, eventually moving back to $A$, and hitting $a_{j}$ first upon return [Meyer 89]. The probability of moving directly from $a_{k}$ to $a_{j}$ in the original chain $\left\{X_{k}\right\}_{k \geq 0}$ is

$$
q_{k j}=\left[\tilde{P}_{11}(\alpha)\right]_{k j}
$$

and the probability of moving directly from $a_{k}$ to $\tilde{a}_{h} \in \tilde{A}$ is

$$
q_{k h}=\left[\tilde{P}_{12}(\alpha)\right]_{k h} .
$$

The probability of moving from $\tilde{a}_{h}$ to $A$ such that $a_{j}$ is the first state entered upon return to $A$ is

$$
\begin{aligned}
q_{h j} & =\sum_{m \geq 0}\left[\tilde{P}_{22}(\alpha)^{m} \tilde{P}_{21}(\alpha)\right]_{h j} \\
& =\left[\left(I-\tilde{P}_{22}(\alpha)\right)^{-1} \tilde{P}_{21}(\alpha)\right]_{h j} .
\end{aligned}
$$

where $\left[\tilde{P}_{22}(\alpha)^{m} \tilde{P}_{21}(\alpha)\right]_{h j}$ is the probability that the original chain starts from the state $\tilde{a}_{h} \in \tilde{A}$, runs exactly $m$ times within $\tilde{A}$, and moves from a state in $\tilde{A}$ to the state $a_{j} \in A$ at the $(m+1)$ th run. Probability $q_{h j}$ can also be obtained by considering the states in $A$ to be absorbing states and applying the 
theory of absorbing chains; see [Kemeny and Snell 76]. Consequently, the onestep transition probability of moving from $a_{k}$ to $a_{j}$ in the new Markov chain $\left\{\phi_{k}\right\}_{k \geq 0}$ is

$$
\begin{aligned}
P\left\{\phi_{n+1}=a_{j} \mid \phi_{n}=a_{k}\right\} & =q_{k j}+\sum_{\tilde{a}_{h} \in \tilde{A}} q_{k h} q_{h j} \\
& =\left[\tilde{P}_{11}(\alpha)+\tilde{P}_{12}(\alpha)\left(I-\tilde{P}_{22}(\alpha)\right)^{-1} \tilde{P}_{21}(\alpha)\right]_{k j} .
\end{aligned}
$$

So, the transition matrix of $\left\{\phi_{k}\right\}_{k \geq 0}$, denoted by $P_{A}(\alpha)$, is formulated as

$$
P_{A}(\alpha)=\tilde{P}_{11}(\alpha)+\tilde{P}_{12}(\alpha)\left(I-\tilde{P}_{22}(\alpha)\right)^{-1} \tilde{P}_{21}(\alpha)
$$

In the theory of Markov chains, $P_{A}(\alpha)$ is called the stochastic complementation of $\tilde{P}_{11}(\alpha)$ [Meyer 89, Stewart 94]. It is known that $P_{A}(\alpha)$ is an irreducible stochastic matrix and has unique stationary distribution. The stationary distribution of $P_{A}(\alpha)$ is

$$
\pi_{A}(\alpha)=\frac{\pi_{1}(\alpha)}{\left\|\pi_{1}(\alpha)\right\|_{1}}
$$

With the above procedure we can construct a return-time Markov chain for each site. We have supposed that there are $N$ sites and $n$ pages in the web graph. The transition matrix $P(\alpha)$ is partitioned into $N \times N$ blocks according to the $N$ sites and has the following form:

$$
P(\alpha)=\left(\begin{array}{cccc}
P_{11}(\alpha) & P_{12}(\alpha) & \cdots & P_{1 N}(\alpha) \\
P_{21}(\alpha) & P_{22}(\alpha) & \cdots & P_{2 N}(\alpha) \\
\vdots & \vdots & \ddots & \vdots \\
P_{N 1}(\alpha) & P_{N 2}(\alpha) & \cdots & P_{N N}(\alpha)
\end{array}\right)
$$

The stationary distribution $\pi(\alpha)$ is partitioned conformally with $P(\alpha)$, i.e.,

$$
\pi(\alpha)=\left(\pi_{1}(\alpha), \pi_{2}(\alpha), \cdots, \pi_{N}(\alpha)\right)
$$

Let $P_{S_{i}}(\alpha)$ denote the transition matrix of the return-time Markov chain for site $S_{i}$ (for $\left.i=1,2, \cdots, N\right)$. From the deduction above, it is clear that the unique stationary distribution of $P_{S_{i}}(\alpha)$ is

$$
\pi_{S_{i}}(\alpha)=\frac{\pi_{i}(\alpha)}{\left\|\pi_{i}(\alpha)\right\|_{1}}, \text { for } i=1,2, \cdots, N
$$


We may conclude that the relation between PageRank and AggregateRank can be formulated as follows. Suppose that AggregateRank is $\xi=\left(\xi_{1}, \xi_{2}, \cdots, \xi_{N}\right)$ and the stationary distribution of $P_{S_{i}}(\alpha)$ is $\pi_{S_{i}}(\alpha), i=1,2, \cdots, N$. Then,

$$
\text { PageRank }=\left(\xi_{1} \pi_{S_{1}}(\alpha), \xi_{2} \pi_{S_{2}}(\alpha), \cdots, \xi_{N} \pi_{S_{N}}(\alpha)\right) .
$$

In other words, the mean frequency of visiting a webpage $i$ belonging to a website $S_{j}$ can be decomposed into two factors: one is the mean frequency of visiting website $S_{j}$, the other is the mean frequency of visiting webpage $i$ when restricted to website $S_{j}$.

Remark 4.I. The above discussions are partly motivated by the stochastic complementation theory and can be regarded as an interesting application of that theory. We refer the reader to [Meyer 89] for a detailed review of stochastic complementation theory.

\section{Algorithm Analysis and Experiments}

In this section, we will discuss the convergence speed and the error bound of the AggregateRank algorithm. Then, some experiments with real web graphs are shown. The engineering aspects of the results of this section were reported in more detail at the 29th Annual International Conference on Research and Development on Information Retrieval [Feng et al. 06].

\section{I. Complexity Analysis}

As can be seen in the previous sections, in our proposed AggregateRank algorithm, we divide the web graph into websites and conduct power-method iterations within each website. After that, we apply the power method once again to the coupling matrix $C^{*}(\alpha)$. It is easy to understand that, in this way, we can save some memory and the corresponding algorithm is easier to implement in parallel. When we deal with a web graph with billions of pages, this advantage will become very meaningful.

However, for the computational complexity, it is not obvious whether the proposed method can be more efficient. The reason is that PageRank has a complexity of $O(r)$. (Suppose that there are $N$ sites, $n$ pages, and $r$ hyperlinks in total, with $r \approx 10 n$.) Considering that $75 \%$ of hyperlinks connect pages in the same website [Henzinger et al. 03], dividing a web graph into websites can save only $25 \%$ of propagations along hyperlinks; thus, the complexity is still 
around $O(r)$. Furthermore, for the computation in Step 5 of the AggregateRank algorithm, it is not obvious whether $C^{*}(\alpha)$ is also a sparse matrix, thus its computational complexity might be as high as $O\left(N^{2}\right)$ in the worse case. All of this can be a big issue.

In this section, we will discuss the aforementioned problems in detail. Specifically, we will prove in Section 5.1.1 that although the complexity of one iteration of the power method applied to $P_{i i}^{*}(\alpha)(i=1, \cdots, N)$ is also $O(r)$, its convergence speed can be significantly faster than that of PageRank over the whole web graph. Then, we will prove in Section 5.1.2 that $C^{*}(\alpha)$ is also a sparse matrix and that there are only about $O(N)$ nonzero elements in this matrix. Therefore, the computation of calculating the stationary distribution for this matrix will also be faster than that for the matrix.

5.I.I. Convergence speed analysis for the power method applied to $P_{i i}^{*}(\alpha)$. In order to understand the convergence speed of the power method applied to $P_{i i}^{*}(\alpha)$, we need to review the following lemma. As we know, the convergence speed of the power method is determined by the magnitude of the subdominant eigenvalue of the transition probability matrix [Stewart 94]. Lemma 5.1 just tells us the relationship between matrix $P(\alpha)$ and its eigenvalues [Langville and Meyer 04].

Lemma 5.I. [Langville and Meyer 04] Given the spectrum of the stochastic matrix $P$ as $\left\{1, \lambda_{2}, \lambda_{3}, \cdots, \lambda_{n}\right\}$, the spectrum of the primitive stochastic matrix

$$
P(\alpha)=\alpha P+(1-\alpha) e v^{T}
$$

is $\left\{1, \alpha \lambda_{2}, \alpha \lambda_{3}, \cdots, \alpha \lambda_{n}\right\}$, where $v^{T}$ is a probability vector.

In order to use Lemma 5.1 to analyze the convergence speed of the power method applied to $P_{i i}^{*}(\alpha)$, we transform $P_{i i}^{*}(\alpha)$ into the following form:

$$
P_{i i}^{*}(\alpha)=\alpha \bar{P}_{i i}^{*}+(1-\alpha) e \frac{e^{T}}{n_{i}},
$$

where $\bar{P}_{i i}^{*}$ is a stochastic matrix and $e^{T} / n_{i}$ is a probability vector.

Given the eigenvalues of $\bar{P}_{i i}^{*}$ as $\left\{1, \tilde{\lambda}_{2}, \tilde{\lambda}_{3}, \cdots, \tilde{\lambda}_{n}\right\}$, by Lemma 5.1 , the eigenvalues of $P_{i i}^{*}(\alpha)$ are $\left\{1, \alpha \tilde{\lambda}_{2}, \alpha \tilde{\lambda}_{3}, \cdots, \alpha \tilde{\lambda}_{n}\right\}$. Since the convergence speed of the power method is determined by the magnitude of the subdominant eigenvalue of $P_{i i}^{*}(\alpha)$, we can conclude that the convergence rate of the power method applied to $P_{i i}^{*}(\alpha)$ is approximately the rate at which $\left(\alpha \tilde{\lambda}_{2}\right)^{k} \rightarrow 0$. 
The convergence speed of PageRank is approximately the rate at which $\alpha^{k} \rightarrow$ 0 . So, whether we can be more efficient than PageRank is determined by how small $\tilde{\lambda}_{2}$ could be. We will show in the following discussions that $\tilde{\lambda}_{2} \ll 1$.

As we know, the web graph has a natural block structure: the majority of hyperlinks are intra-site links [Kamvar et al. 03]. Therefore, a random walk on the web with the transition matrix $P(\alpha)$ can be viewed as a nearly completely decomposable Markov chain. According to [Meyer 89], when the states in a nearly completely decomposable Markov chain are naturally ordered, and when the transition matrix is partitioned into $N$ closely coupled subclasses in the natural way, the underlying transition matrix of the Markov chain has exactly $N-1$ non-unit eigenvalues clustered near $\lambda=1$. (There are pathological cases, but they are rare in practical work [Meyer 89].) Thus, $P(\alpha)$ has exactly $N-1$ non-unit eigenvalues clustered near $\lambda=1$.

Since $P_{i i}^{*}(\alpha)$ is an irreducible stochastic matrix, the Perron-Frobenius theorem [Gantmacher 59] guarantees that the unit eigenvalue of each $P_{i i}^{*}(\alpha)$ is simple. Because $P_{i i}^{*}(\alpha) \approx P_{i i}(\alpha)$, by the continuity of the eigenvalues, the nonunit eigenvalues of $P_{i i}^{*}(\alpha)$ must be rather far from the unit eigenvalue of $P_{i i}^{*}(\alpha)$. Otherwise, the spectrum of $P(\alpha)$ would contain a cluster of at least $N$ nonunit eigenvalues positioned near $\lambda=1$. As a result, we can come to the conclusion that $\alpha \tilde{\lambda}_{2} \ll 1$ for any $\alpha$ close to 1 ; thus, $\tilde{\lambda}_{2} \ll 1$. That is, the convergence speed of the power method applied to $P_{i i}^{*}(\alpha)$ is much faster than that of PageRank.

5.I.2. Complexity of the power method applied to $C^{*}(\alpha)$. As mentioned at the beginning of Section 5 , the sparseness of the matrix $C^{*}(\alpha)$ is a critical factor that influences the computational complexity of our proposed AggregateRank algorithm. To understand this, we conduct the following discussions. First of all, we transform $P(\alpha)$ into the following form:

$$
\begin{aligned}
P(\alpha) & =\alpha \bar{P}+(1-\alpha) e \frac{e^{T}}{n} \\
& =\alpha\left(P+a \frac{1}{n} e^{T}\right)+(1-\alpha) e \frac{e^{T}}{n} \\
& =\alpha P+(\alpha a+(1-\alpha) e) \frac{e^{T}}{n},
\end{aligned}
$$

where $P$ is the transition matrix whose element $p_{i j}$ is the probability of moving from webpage $i$ to webpage $j$ in one step following the hyperlink structure of the web graph, $a$ is a vector whose element $a_{i}=1$ if row $i$ of $P$ corresponds to a dangling node and equals 0 otherwise, $\alpha$ is damping factor, and $e$ is a column vector of all ones. Then, we investigate the construction process of $C^{*}(\alpha)$ as 
follows:

$$
\begin{aligned}
C^{*}(\alpha) & =U(\alpha) P(\alpha) V \\
& =U(\alpha)\left(\alpha P+(\alpha a+(1-\alpha) e) \frac{1}{n} e^{T}\right) V \\
& =\alpha U(\alpha) P V+(\alpha U(\alpha) a+(1-\alpha) e) v^{T}
\end{aligned}
$$

where

$$
\begin{gathered}
U(\alpha)=\left(\begin{array}{llll}
u_{1}(\alpha) & & & \\
& u_{2}(\alpha) & & \\
& & \ddots & \\
& & & u_{N}(\alpha)
\end{array}\right)_{N \times n}, \\
V=\left(\begin{array}{llll}
e & & & \\
& e & & \\
& & \ddots & \\
& & & e
\end{array}\right)_{n \times N},
\end{gathered}
$$

and $v^{T}=\left(\frac{n_{1}}{n}, \frac{n_{2}}{n}, \cdots, \frac{n_{N}}{n}\right)$ is a probability vector.

According to this decomposition, in Step 4 of the AggregateRank algorithm, we only need to compute $A=: U(\alpha) P V$. The corresponding count of multiplications is $O(r)$. Note that we do not need any iteration here, so the complexity of Step 4 is actually much lower than that of PageRank, which will take tens or even hundreds of iterations to converge.

In Step 5, for any starting vector $\xi^{*(0)}(\alpha)$,

$$
\begin{aligned}
\xi^{*(k)}(\alpha) & =\xi^{*(k-1)}(\alpha) C^{*}(\alpha) \\
& =\xi^{*(k-1)}(\alpha) U(\alpha) P(\alpha) V \\
& =\xi^{*(k-1)}(\alpha) U(\alpha)\left(\alpha\left(P+a \frac{1}{n} e^{T}\right)+(1-\alpha) \frac{1}{n} e e^{T}\right) V \\
& =\alpha \xi^{*(k-1)} A+\left(\alpha \xi^{*(k-1)}(\alpha) U(\alpha) a+(1-\alpha)\right) v^{T} .
\end{aligned}
$$

Then, it is clear that the computational complexity of each iteration in Step 5 depends on the number of nonzeroes in $A$. Because $a_{i j}$ is equal to the linear combination of the elements in block $P_{i j}$, we have $a_{i j}=0$ when every element in $P_{i j}$ is 0 . Suppose that the average number of sites to which a particular website links is $\mu$; then, $A$ has $\mu$ nonzeroes in each row, and the number of nonzeroes in $A$ is $\mu N$. Considering that, for the World Wide Web, $\mu$ is almost a constant which is ten or so [Bharat et al. 01], we come to the conclusion that the computational complex of one iteration in Step 5 is $O(N) \ll O(r)$. 


\subsection{Error Analysis}

As one can see, the proposed AggregateRank algorithm is an approximation to PageRankSum. In this subsection, we will discuss the error bound of this approximation.

According to the theory of stochastic complement, the approximation shown in (3.13) requires the matrix to be nearly completely decomposable. This condition is well satisfied in real web applications, because about $75 \%$ of the hyperlinks connect pages in the same website [Henzinger et al. 03], and it is reasonable to treat the transition probability matrix $P(\alpha)$ as a nearly completely decomposable matrix.

According to the discussions in Section 5.1.1, $P(\alpha)$ has exactly $N-1$ non-unit eigenvalues that are very close to the unit eigenvalue. Thus, the approximation in (3.13) has an upper error bound according to [Cho and Meyer], which is determined by the number of pages $n$, the number of sites $N$, the size of each site $n_{i}$, and the condition number of the coupling matrix $\kappa(C(\alpha))$, which is the deviation from the complete reducibility $\delta$ and the eigen structure of the probability transition matrix $P(\alpha)$. We state it explicitly in the following theorem.

Theorem 5.2. If $P(\alpha)$ has exactly $N-1$ non-unit eigenvalues close to the unit eigenvalue, then there are the following error bounds:

$$
\begin{array}{r}
\left\|\frac{\pi_{i}(\alpha)}{\left\|\pi_{i}(\alpha)\right\|_{1}}-u_{i}(\alpha)\right\|_{1} \leq \min \left\{\delta\left(n_{i}-1\right), 1\right\}, \\
\left\|C(\alpha)-C^{*}(\alpha)\right\|_{\infty} \leq \min \left\{\delta^{2}\left(\max _{i} n_{i}-1\right), 1\right\}, \\
\left\|\xi(\alpha)-\xi^{*}(\alpha)\right\|_{1} \leq \min \left\{\delta^{2} \kappa(C(\alpha))\left(\max _{i} n_{i}-1\right), 1\right\} .
\end{array}
$$

From Theorem 5.2, we can see that the upper error bound of the AggregateRank algorithm principally depends on the scale of the largest website. Evidently, the number of pages in the largest website is much smaller than the size of the web. In this regard, we can say that the corresponding error bound is well controlled.

\subsection{Experiments}

In our experiments, the data corpus is the benchmark data for the web track of TREC 2003 and 2004, which was crawled from the gov domain in the year 2002. It contains $1,247,753$ webpages in total. 


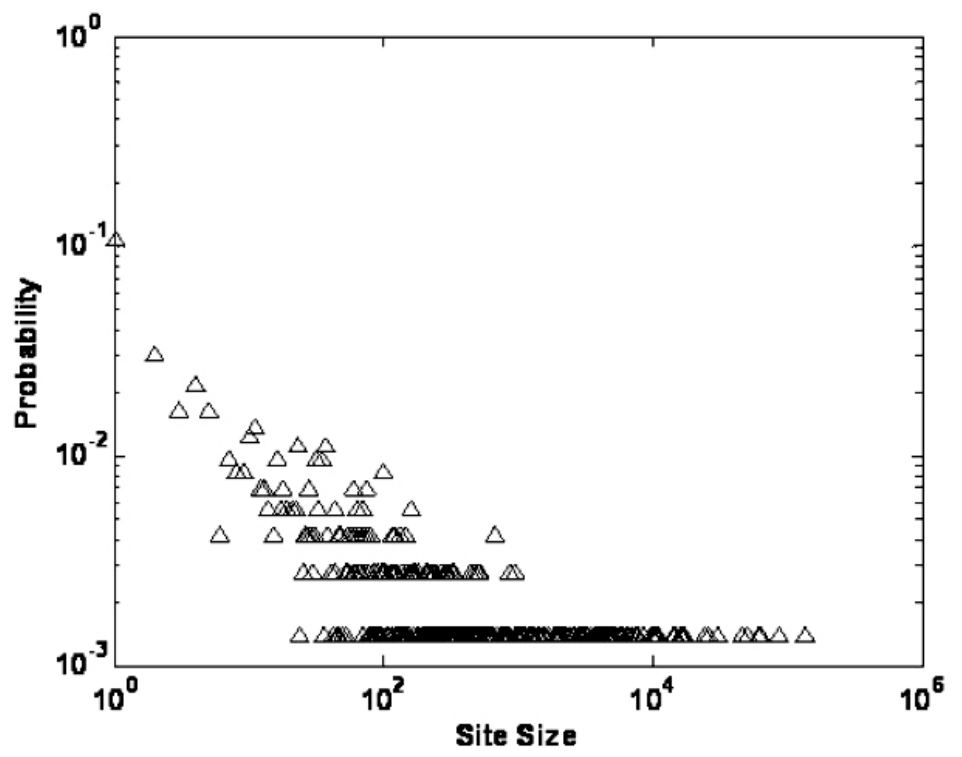

Figure 3. The distribution of the sizes of .gov websites.

Before testing our proposed method, we needed to partition the web graph into websites. For this purpose, we followed the rules as below. Because the URLs in the gov domain are very regular, we can easily determine the website to which a webpage belongs. After removing the http:// or https:// from the URL, the part before the first slash can be considered as the website name. However, because there may be some subsites, we only use the word preceding .gov as the identifier for the site. For example, http://aaa.bbb.gov/xxxx belongs to the website bbb.

After this preprocess, we had 731 sites in the gov dataset. The largest website contained 137,103 webpages while the smallest one contained only one page. The distribution of the sizes of all the websites is shown in Figure 3. It nearly follows a power law and is consistent with previous research on the sizes of websites [Albert and Barabasi 02].

In our experiment, we validated whether the proposed AggregateRank algorithm can well approximate PageRankSum. For comparison, we also investigated two other HostRank algorithms, which work on the weighted hostgraph and naïve hostgraph respectively [Bharat et al. 01, Dill et al. 01]. The differences between PageRankSum and the algorithms under investigation are shown in Table 1, in terms of the Euclidean distance between the rank vectors.

From Table 1, we can see that the AggregateRank algorithm has the best performance: its Euclidean distance from PageRankSum is only 0.0057, while 


\begin{tabular}{|c|c|c|c|}
\hline $\begin{array}{c}\text { Ranking } \\
\text { algorthms }\end{array}$ & $\begin{array}{c}\text { Euclidean } \\
\text { distance }\end{array}$ & $\begin{array}{c}\text { Max distance in } \\
\text { single dimension }\end{array}$ & $\begin{array}{c}\text { Min distance in } \\
\text { single dimension }\end{array}$ \\
\hline PageRankSum & 0.0 & 0.0 & 0.0 \\
\hline AggregateRank & 0.0057 & 0.0029 & 0.000000 \\
\hline weighted HostRank & 0.1125 & 0.0805 & 0.000020 \\
\hline naïve HostRank & 0.1601 & 0.1098 & 0.000007 \\
\hline
\end{tabular}

Table I. Performance evaluation of algorithms based on Euclidean distance.

the ranking results produced by the other two algorithms are farther from PageRankSum, with Euclidean distances of 0.1125 and 0.1601 , respectively.

In addition to the Euclidean distance, we also used another similarity measure based on the Kendall's $\tau$ distance [Kendall and Gibbons 90] to evaluate the performance of the ranking results. This measure ignores the absolute values of the ranking scores and only counts the partial-order preferences. Therefore, it can better reflect the true ranking performance in real applications. This similarity between two ranking lists $s$ and $t$ is defined as follows:

$$
\operatorname{Sim}(s, t)=1-\frac{K(s, t)}{C_{n}^{2}}
$$

where $K(s, t)$ is the Kendall's $\tau$ distance, which counts the number of pair-wise disagreements between $s$ and $t$, defined as

$$
K(s, t)=|(i, j)| i<j, s(i)<s(j), t(i)>t(j) \mid .
$$

According to the definition, the larger $\operatorname{Sim}(s, t)$ is, the more similar two lists are. If the two ranking lists are consistent with each other, their Sim measure is equal to 1 .

We list the performance evaluation of the aforementioned algorithms based on the Kendall's $\tau$ distance in Table 2. From this table, once again we can see that the AggregateRank algorithm is the best approximation to PageRankSum. Furthermore, the advantage of the AggregateRank algorithm over the reference algorithms becomes even more obvious if we look at the top- $k$ ranking results.

\begin{tabular}{|c|c|}
\hline Ranking algorithms & Sim \\
\hline PageRankSum & 1 \\
\hline AggregateRank & 0.9826 \\
\hline weighted HostRank & 0.8428 \\
\hline naïve HostRank & 0.8889 \\
\hline
\end{tabular}

Table 2. Performance evaluation of ranking algorithms based on Kendall's $\tau$ distance. 


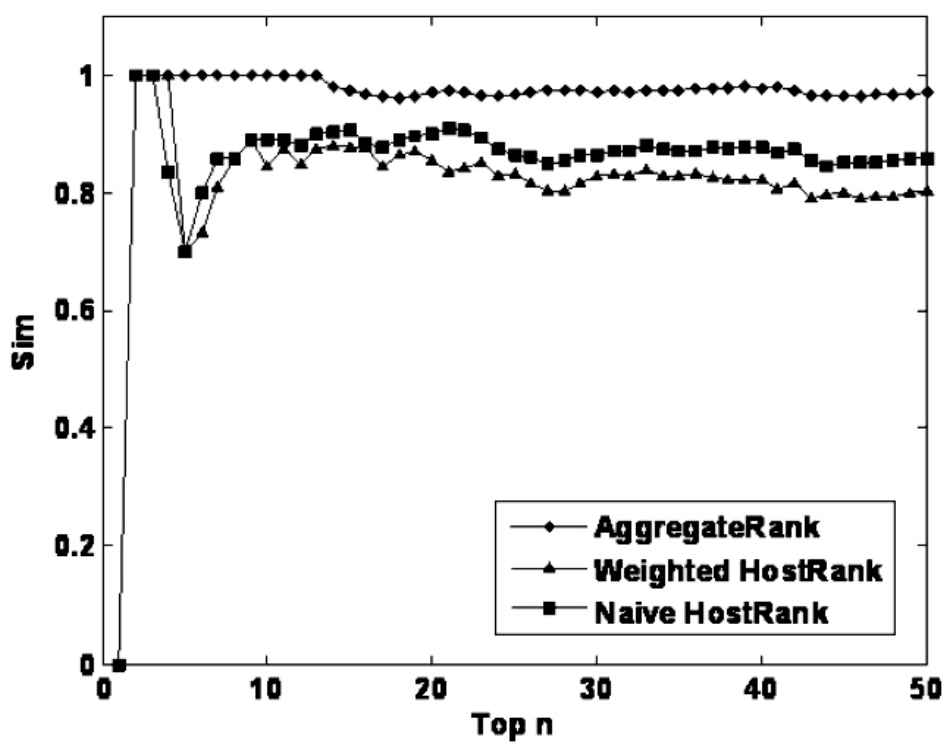

Figure 4. Similarity between PageRankSum and the three other ranking results.

Actually, we took the top $k$ websites in terms of their PageRankSum and obtained their order $O_{p}$. Then, we calculated their relative orders according to the other ranking algorithms discussed, i.e. $O_{a}, O_{w}$, and $O_{n}$, which correspond to the AggregateRank algorithm, the weighted HostRank algorithm, and the naïve HostRank algorithm, respectively. We plot the similarity based on the Kendall's $\tau$ distance between these top- $k$ ranking results in Figure 4.

After the comparison on similarity, we compared these ranking algorithms based on complexity as well. As discussed in Section 3, the AggregateRank algorithm can converge faster than PageRank. To verify this, we use the $L_{1}$-norm of the difference between the current ranking list and the last one to measure whether the power method converges. When this difference is less than $10^{-3}$, we regard the computation as converged, and the computation process is terminated. The running time of each algorithm is shown in Table 3.

\begin{tabular}{|c|c|}
\hline Ranking algorithms & Running time(s) \\
\hline PageRankSum & 116.23 \\
\hline AggregateRank & 29.83 \\
\hline Weighted HostRank & 0.22 \\
\hline naïve HostRank & 0.10 \\
\hline
\end{tabular}

Table 3. Comparison of the running times of the four algorithms. 
From Table 3, we can see that the proposed AggregateRank method is faster than PageRankSum, while a little more complex than the HostRank methods. This is consistent with the theoretical analysis in the previous section. The fast speed of AggregateRank mainly comes from the fast convergence speed. And the fast speed of HostRank comes from the low dimension of the hostgraph.

In summary, by taking effectiveness and efficiency into consideration at the same time, we consider the proposed AggregateRank algorithm to be a better solution to website ranking.

Acknowledgments. The work is partly supported by the National Natural Science Foundation of China, 973 Project, and the Science and Practice Fund for Graduate of Chinese Academy of Sciences.

\section{References}

[Albert and Barabasi 02] R. Albert and A.-L. Barabasi. "Statistical Mechanics of Complex Networks." Rev. Mod. Phys. 74:1 (2002), 47-97.

[Bao and Liu 06] Y. Bao and Y. Liu. "Limit of PageRank with Damping Factor." Dynamics of Continuous, Discrete and Impulsive Systems, Series B 13:3 (2006), 497-504.

[Bharat et al. 01] K. Bharat, B.-W. Chang, M. Henzinger, and M. Ruhl. "Who Links to Whom: Mining Linkage between Web Sites." In Proceedings of the 2001 IEEE International Conference on Data Mining, pp. 51-58. Los Alamitos, CA: IEEE Computer Society, 2001.

[Brin et al. 99] S. Brin, L. Page, R. Motwami, and T. Winograd. "The PageRank Citation Ranking: Bringing Order to the Web." Technical Report 1999-0120, Computer Science Department, Stanford University, 1999.

[Cho and Meyer 06] G. E. Cho and C. D. Meyer. "Aggregation/Disaggregation Methods of Nearly Uncoupled Markov Chains." Preprint, 2006. Available from World Wide Web (http://meyer.math.ncsu.edu/Meyer/PS_Files/Numcad.ps).

[Davulcu et al. 04] H. Davulcu, S. Vadrevu, and S. Nagarajan. "OntoMiner: Bootstrapping Ontologies from Overlapping Domain Specific Web Sites." In Proceedings of the 13th International World Wide Web Conference on Alternate Track Papers 63 Posters, pp. 500-501. New York: ACM Press, 2004.

[Despeyroux 04] T. Despeyroux. "Practical Semantic Analysis of Web Sites and Documents." In Proceedings of the 13th International Conference on World Wide Web, pp. 685-693. New York: ACM Press, 2004.

[Dill et al. 01] S. Dill, R. Kumar, K. McCurley, S. Rajagopalan, D. Sivakumar, and A. Tomkins. "Self-Similarity in the Web." In Proceedings of 27th International Conference on Very Large Data Bases, pp. 69-78. San Francisco: Morgan Kaufmann, 2001. 
[Eiron et al. 04] N. Eiron, K. S. McCurley, and J. A. Tomlin. "Ranking the Web Frontier." In Proceedings of the 13th International Conference on World Wide Web, pp. 309-318. New York: ACM Press, 2004.

[Feng et al. 06] Guang Feng, Tie-Yan Liu, Ying Wang, Ying Bao, Zhi-Ming Ma, XuDong Zhang, and Wei-Ying Ma. "AggrerateRank: Bringing Order to Web Sites." In Proceedings of the 29th Annual International ACM SIGIR Conference on Research and Development in Information Retrieval, pp. 75-82. New York: ACM Press, 2006.

[Gantmacher 59] F. R. Gantmacher. Matrix Theory, Volume 2. New York: Chelsea, 1959.

[Girvan and Newman 02] M. Girvan and M. E. J. Newman. "Community Structure in Social and Biological Networks." Proc. Natl. Acad. Sci. 99:12 (2002), 7821-7826.

[Henzinger et al. 03] M. R. Henzinger, R. Motwani, and C. Silverstein. "Challenges in Web Search Engines." In Proceedings of the Eighteenth International Joint Conference on Artificial Intelligence, pp. 1573-1579. San Francisco: Morgan Kaufmann, 2003.

[Kallenberg 97] O. Kallenberg. Foundations of Modern Probability. New York: Springer-Verlag, 1997.

[Kamvar et al. 03] S. Kamvar, T. Haveliwala, C. Manning, and G. Golub. "Exploiting the Block Structure of the Web for Computing PageRank." Technical report, Stanford University, 2003.

[Kemeny and Snell 76] J. Kemeny and J. L. Snell. Finite Markov Chains. New York: Springer-Verlag, 1976.

[Kendall and Gibbons 90] M. Kendall and J. Gibbons. Rank Correlation Methods, Fifth edition. London: Edward Arnold, 1990.

[Kleinberg 99] J. Kleinberg. "Authoritative Sources in a Hyperlinked Environment." J.ACM 46 (1999), 604-632.

[Langville and Meyer 04] A. N. Langville and C. D. Meyer. "Deeper inside PageRank." Internet Mathematics 1:3 (2004), 355-400.

[Lei et al. 04] Y. Lei, Enrico Motta, and John Domingue. "Modelling Data-Intensive Web Sites with OntoWeaver." In Proceedings of the International Workshop on Web Information Systems Modeling, Riga, Latvia, 2004. Available from World Wide Web (http://kmi.open.ac.uk/projects/akt/ontoweaver/ resources/publications/OntoWeaver_WISM2004_v2.pdf), 2004.

[Lerman et al. 04] K. Lerman, L. Getoor, S. Minton, and C. Knoblock. "Using the Structure of Web Sites for Automatic Segmentation of Tables." In Proceedings of the 2004 ACM SIGMOD International Conference on Management of Data, pp. 119-130. New York: ACM Press, 2004.

[Meyer 89] C. D. Meyer. "Stochastic Complementation, Uncoupling Markov Chains, and the Theory of Nearly Reducible Systems." SIAM Review 31:2 (1989), 240-272.

[Qian and Gong 97] M. Qian and G. Gong. Theory of Stochastic Processes, Second edition (in Chinese). Beijing: Peking University Press, 1997. 
[Qin et al. 05] T. Qin, T. Y. Liu, X. D. Zhang, G. Feng, and W. Y. Ma. "Subsite Retrieval: A Novel Concept for Topic Distillation." In Information Retrieval Technology: Second Asia Information Retrieval Symposium, Jeju Island, Korea, October 13-15, 2005, Proceedings, pp. 388-400, Lecture Notes in Computer Science 3689. Berlin: Springer, 2005.

[Stewart 94] W. J. Stewart. Introduction to the Numerical Solution of Markov Chains. Princeton: Princeton University Press, 1994.

[Wu and Aberer 04] J. Wu and K. Aberer. "Using SiteRank for P2P Web Retrieval." EPFL Technical Report ID: IC/2004/31, 2004.

Ying Bao, Academy of Mathematics and Systems Science, Chinese Academy of Sciences, Beijing, 100080, PR China (ybao@amss.ac.cn)

Guang Feng, Department of Electronic Engineering, Tsinghua University, Beijing, 100084, PR China (fengg03@mails.tsinghua.edu.cn)

Tie-Yan Liu, Microsoft Research Asia, 4F, Sigma Center, No. 49, Zhichun Road, Haidian District, Beijing, 100080, PR China (tie-yan.liu@microsoft.com)

Zhi-Ming Ma, Academy of Mathematics and Systems Science, Chinese Academy of Sciences, Beijing, 100080, PR China (mazm@amt.ac.cn)

Ying Wang, Academy of Mathematics and Systems Science, Chinese Academy of Sciences, Beijing, 100080, PR China (wangying@amss.ac.cn)

Received October 20, 2006; accepted December 27, 2006. 